\title{
A CHARACTERIZATION OF THE DISCONTINUITY POINT SET OF STRONGLY SEPARATELY CONTINUOUS FUNCTIONS ON PRODUCTS
}

\author{
OLENA KARLOVA* AND VOLODYMYR MYKHAYLYUK**
}

\begin{abstract}
We study properties of strongly separately continuous mappings defined on subsets of products of topological spaces equipped with the topology of pointwise convergence. In particular, we give a necessary and sufficient condition for a strongly separately continuous mapping to be continuous on a product of an arbitrary family of topological spaces. Moreover, we characterize the discontinuity point set of strongly separately continuous function defined on a subset of countable product of finite-dimensional normed spaces.
\end{abstract}

\section{INTRODUCTION}

In 1998 Omar Dzagnidze [1 introduced a notion of a strongly separately continuous function $f: \mathbb{R}^{n} \rightarrow \mathbb{R}$. Namely, he calls a function $f$ strongly separately continuous at a point $x^{0}=\left(x_{1}^{0}, \ldots, x_{n}^{0}\right) \in \mathbb{R}^{n}$ if for every $k=1, \ldots, n$ the equality $\lim _{x \rightarrow x^{0}}\left|f\left(x_{1}, \ldots, x_{k}, \ldots, x_{n}\right)-f\left(x_{1}, \ldots, x_{k}^{0}, \ldots, x_{n}\right)\right|=0$ holds. Dzagnidze proved that a function $f: \mathbb{R}^{n} \rightarrow \mathbb{R}$ is strongly separately continuous at $x^{0}$ if and only if $f$ is continuous at $x^{0}$.

Extending the investigations of Dzagnidze, J. Činčura, T. Šalát and T. Visnyai in 2] consider strongly separately continuous functions defined on the space $\ell_{2}$ of sequences $x=\left(x_{n}\right)_{n=1}^{\infty}$ of real numbers such that $\sum_{n=1}^{\infty} x_{n}^{2}<+\infty$ endowed with the standard metric $d(x, y)=\left(\sum_{n=1}^{\infty}\left(x_{n}-y_{n}\right)^{2}\right)^{1 / 2}$. In particular, the authors gave an example of a strongly separately continuous everywhere discontinuous function $f: \ell_{2} \rightarrow \mathbb{R}$.

Recently, T. Visnyai in [3] continued to study properties of strongly separately continuous functions on $\ell_{2}$ and constructed a strongly separately continuous

2010 Mathematics Subject Classification. Primary 54C08, 54C30; Secondary 26B05.

Key words and phrases. strongly separately continuous function, separately continuous function. 
function $f: \ell_{2} \rightarrow \mathbb{R}$ which belongs to the third Baire class and is not quasicontinuous at every point. Moreover, T. Visnyai gave a sufficient condition for strongly separately continuous function to be continuous on $\ell_{2}$.

In this paper we study strongly separately continuous functions defined on subsets of a product $\prod_{t \in T} X_{t}$ of topological spaces $X_{t}$ equipped with the Tychonoff topology of pointwise convergence. In Section 2 we introduce a notion and give the simplest properties of $\mathcal{S}$-topology which is tightly connected with strongly separately continuous functions. In the third section we give a necessary and sufficient condition for a strongly separately continuous mapping to be continuous on a product of an arbitrary family of topological spaces. In Section 4 we find a necessary condition on a set to be the discontinuity point set of a strongly separately continuous mapping defined on a product of topological spaces. Finally, in the fifth section we describe the discontinuity point set of a strongly separately continuous function defined on a subset of a countable product of finite-dimensional normed spaces.

\section{A Notion AND PROPERTIES OF $\mathcal{S}$-TOPOLOGY}

Let $X=\prod_{t \in T} X_{t}$ be a product of a family of sets $X_{t}$ with $\left|X_{t}\right|>1$ for all $t \in T$. If $S \subseteq S_{1} \subseteq T, a=\left(a_{t}\right)_{t \in T} \in X, x=\left(x_{t}\right)_{t \in S_{1}} \in \prod_{t \in S_{1}} X_{t}$, then we denote by $a_{S}^{x}$ a point $\left(y_{t}\right)_{t \in T}$, where

$$
y_{t}= \begin{cases}x_{t}, & t \in S \\ a_{t}, & t \in T \backslash S .\end{cases}
$$

In the case $S=\{s\}$ we shall write $a_{s}^{x}$ instead of $a_{\{s\}}^{x}$.

If $n \in \mathbb{N}$, then

$$
\sigma_{n}(x)=\left\{y=\left(y_{t}\right)_{t \in T} \in X:\left|\left\{t \in T: y_{t} \neq x_{t}\right\}\right| \leq n\right\}
$$

and

$$
\sigma(x)=\bigcup_{n=1}^{\infty} \sigma_{n}(x) .
$$

Each of the sets of the form $\sigma(x)$ for an $x \in X$ is called $a \sigma$-product of $X$.

We denote by $\tau$ the Tychonoff topology on a product $X=\prod_{t \in T} X_{t}$ of topological spaces $X_{t}$. If $X_{0} \subseteq X$, then the symbol $\left(X_{0}, \tau\right)$ means the subspace $X_{0}$ equipped with the Tychonoff topology induced from $(X, \tau)$.

If $X_{t}=X$ for all $t \in T$ then the product $\prod_{t \in T} X_{t}$ we also denote by $X^{\mathfrak{m}}$, where $\mathfrak{m}=|T|$.

Definition 2.1. A set $A \subseteq \prod_{t \in T} X_{t}$ is called $\mathcal{S}$-open if $\sigma_{1}(x) \subseteq A$ for all $x \in A$. 
We remark that the definition of an $\mathcal{S}$-open set develops the definition of a set of the type $\left(P_{1}\right)$ introduced in [2].

Let $\mathcal{S}(X)$ denote the collection of all $\mathcal{S}$-open subsets of $X$. We notice that $\mathcal{S}(X)$ is a topology on $X$. We will denote by $(X, \mathcal{S})$ the product $X=\prod_{t \in T} X_{t}$ equipped with the topology $\mathcal{S}(X)$.

The proof of the following is straightforward.

Proposition 2.1. Let $X=\prod_{t \in T} X_{t},\left|X_{t}\right|>1$ for all $t \in T, x \in X$ and $A \subseteq X$. Then

(1) $A \in \mathcal{S}(X)$ if and only if $X \backslash A \in \mathcal{S}(X)$;

(2) $A \in \mathcal{S}(X)$ if and only if $A=\bigcup_{x \in A} \sigma(x)$;

(3) $\sigma(x)$ is the smallest $\mathcal{S}$-open set which contains $x$;

(4) if $A \in \mathcal{S}(X)$, then $A$ is dense in $(X, \tau)$.

(5) there exists a non-trivial $\mathcal{S}$-open subset of $X$ if and only if $|T| \geq \aleph_{0}$.

It follows from Proposition 2.1 that $\sigma$-products of two distinct points of $\prod_{t \in T} X_{t}$ either coincide, or does not intersect. Consequently, the family of all $\sigma$-products of an arbitrary $\mathcal{S}$-open set $X \subseteq \prod_{t \in T} X_{t}$ generates a partition of $X$ on mutually disjoint $\mathcal{S}$-open sets, which we will call $\mathcal{S}$-components of $X$.

Notice that every $\mathcal{S}$-component of $X$ is an indiscrete subspace of $(X, \mathcal{S}(X))$ and the space $(X, \mathcal{S}(X))$ is a topological sum of indiscrete spaces.

Proposition 2.1 easily implies the following properties of the $\mathcal{S}$-topology.

Proposition 2.2. Let $X=\prod_{t \in T} X_{t}$ and $\left|X_{t}\right|>1$ for all $t \in T$. Then the space $(X, \mathcal{S})$

(1) is first-countable;

(2) does not satisfy $T_{0}, T_{1}$ and $T_{2}$ if $T \neq \emptyset$;

(3) satisfy $T_{3}, T_{3 \frac{1}{2}}$ and $T_{4}$;

(4) is arcwise connected if and only if $|T|<\aleph_{0}$;

(5) is extremally disconnected.

3. A NECESSARY AND SUFFICIENT CONDITION FOR A STRONGLY SEPARATELY CONTINUOUS MAPPING TO BE CONTINUOUS

Definition 3.1. Let $\left(X_{t}: t \in T\right)$ be a family of topological spaces, $Y$ be a topological space and let $X \subseteq \prod_{t \in T} X_{t}$ be an $\mathcal{S}$-open set. A mapping $f: X \rightarrow Y$ is said to be separately continuous at a point $a=\left(a_{t}\right)_{t \in T} \in X$ with respect to the $t$-th variable provided that the mapping $g: X_{t} \rightarrow Y$ defined by the rule $g(x)=f\left(a_{t}^{x}\right)$ for all $x \in X_{t}$ is continuous at the point $a_{t} \in X_{t}$. 
Let $f: X \rightarrow Y$ be a mapping between topological spaces $X$ and $Y, a \in X$ and $b \in Y$. Then $\lim _{x \rightarrow a} f(x)=b$ if and only if for any neighborhood $V$ of $b$ in $Y$ there is a neighborhood $U$ of $a$ in $X$ such that $f(U) \subseteq V$.

Definition 3.2. Let $X \subseteq \prod_{t \in T} X_{t}$ be an $\mathcal{S}$-open set, $\mathcal{T}$ be a topology on $X$ and let $(Y, d)$ be a metric space. A mapping $f:(X, \mathcal{T}) \rightarrow Y$ is called strongly separately continuous at a point $a \in X$ with respect to the $t$-th variable if

$$
\lim _{x \rightarrow a} d\left(f(x), f\left(x_{t}^{a}\right)\right)=0 .
$$

Definition 3.3. A mapping $f: X \rightarrow Y$ is

- (strongly) separately continuous at a point $a \in X$ if $f$ is (strongly) separately continuous at $a$ with respect to each variable $t \in T$;

- (strongly) separately continuous on the set $X$ if $f$ is (strongly) separately continuous at each point $a \in X$ with respect to each variable $t \in T$.

Definition 3.4. A set $A \subseteq \prod_{t \in T} X_{t}$ is called projectively symmetric with respect to a point $a \in A$ if for all $t \in T$ and for all $x \in A$ we have $x_{t}^{a} \in A$.

Definition 3.5. Let $X \subseteq \prod_{t \in T} X_{t}$ and $\mathcal{T}$ be a topology on $X$. Then $(X, \mathcal{T})$ is said to be locally projectively symmetric if every $x \in X$ has a base of projectively symmetric neighborhoods with respect to $x$.

It is easy to see that an arbitrary $\mathcal{S}$-open subset of a product $\prod_{t \in T} X_{t}$ of topological spaces $X_{t}$ equipped either with the Tychonoff topology $\tau$, or with the $\mathcal{S}$-topology, is a locally projectively symmetric space. All classical spaces of sequences as the space $c$ of all convergence sequences or the spaces $\ell_{p}$ with $0<p \leq \infty$ are locally projectively symmetric.

Proposition 3.1. Let $X$ be an $\mathcal{S}$-open subset of a product $\prod_{t \in T} X_{t}$ of topological spaces equipped with a locally projectively symmetric topology $\mathcal{T}, a=\left(a_{t}\right)_{t \in T} \in$ $X, Y$ be a metric space and let $f:(X, \mathcal{T}) \rightarrow Y$ be a continuous mapping at the point a. Then $f$ is strongly separately continuous at $a$.

Proof. Fix $\varepsilon>0$ and $t \in T$. Take a projectively symmetric with respect to $a$ neighborhood $U$ of $a$ such that

$$
d(f(x), f(a))<\frac{\varepsilon}{2}
$$

for all $x \in U$. Then $x_{t}^{a} \in U$ and

$$
d\left(f(x), f\left(x_{t}^{a}\right)\right) \leq d(f(x), f(a))+d\left(f(a), f\left(x_{t}^{a}\right)\right)<\frac{\varepsilon}{2}+\frac{\varepsilon}{2}=\varepsilon
$$

for all $x \in U$. 
Note that the inverse implication is not true in case $(X, \mathcal{T})$ is not a locally projectively symmetric space, as the example below shows.

Example 1. Let $X$ be the Niemytski plane, i.e. $X=\mathbb{R} \times[0,+\infty)$, where a base of neighborhoods of $(x, y) \in X$ with $y>0$ form open balls with the center in $(x, y)$, and a base of neighborhoods of $(x, 0)$ form the sets $U \cup\{(x, 0)\}$, where $U$ is an open ball which tangents to $\mathbb{R} \times\{0\}$ in the point $(x, 0)$. Then there exists a continuous function $f: X \rightarrow \mathbb{R}$, which is not strongly separately continuous.

Proof. Denote $a=(0,0)$ and consider an arbitrary basic neighborhood $U$ of $a$ in $X$. Since $X$ is a completely regular space, there exists a continuous function $f: X \rightarrow[0,1]$ such that $f(a)=0$ and $f(x, y)=1$ for all $(x, y) \in X \backslash U$. We show that $f$ is not strongly separately continuous at the point $a$ with respect to the second variable. Indeed, fix $\varepsilon=\frac{1}{2}$ and a neighborhood $V$ of $a$. By the continuity of $f$ at $a$ we choose a neighborhood $V_{0}$ of $a$ such that $V_{0} \subseteq V$ and $f(x, y)<\frac{1}{2}$ for all $(x, y) \in V_{0}$. Now let $(x, y) \in V_{0}$ be an arbitrary point with $x \neq 0$. Then $f(x, 0)=1$ and $|f(x, y)-f(x, 0)|=1-f(x, y)>\frac{1}{2}$. Hence, $f$ is not strongly separately continuous.

Let $\mathcal{E}$ denote the Euclidean topology on $\mathbb{R}, X_{1}=X_{2}=(\mathbb{R}, \mathcal{E})$ and let $\mathcal{T}$ be a discrete topology on the set $X=\mathbb{R} \times \mathbb{R}$. We consider the following function $f: X \rightarrow \mathbb{R}$

$$
f\left(x_{1}, x_{2}\right)= \begin{cases}1, & \left(x_{1}, x_{2}\right) \in X \backslash\{(0,0)\} \\ 0, & \left(x_{1}, x_{2}\right)=(0,0)\end{cases}
$$

Then $f$ is continuous on $(X, \mathcal{T})$, since $X$ is discrete, but $f$ is not separately continuous on $X_{1} \times X_{2}$ at the point $(0,0)$ by definition 3.1. Therefore, it is natural to find conditions on a topology on a product of topological spaces such that the implication " $f$ is continuous $\Rightarrow f$ is separately continuous" holds.

Definition 3.6. Let $X \subseteq \prod_{t \in T} X_{t}$ be an $\mathcal{S}$-open subset of a product of topological spaces $\left(X_{t}, \mathcal{T}_{t}\right)$ and let $\mathcal{T}$ be a topology on $X$. We say that $\mathcal{T}$ is coordinated with the family $\left(\mathcal{T}_{t}: t \in T\right)$ if

$$
\lim _{x \rightarrow a_{t}} a_{t}^{x}=a
$$

for all $t \in T$ and $a=\left(a_{s}\right)_{s \in T} \in X$.

Proposition 3.2. Let $\mathcal{T}$ be a topology on an $\mathcal{S}$-open subset $X$ of a product of topological spaces $\left(X_{t}, \mathcal{T}_{t}\right)$ coordinated with the family $\left(\mathcal{T}_{t}: t \in T\right), Y$ is a (metric) space and $f: X \rightarrow Y$ be a (strongly separately) continuous mapping at $a$ point $a \in X$. Then $f$ is separately continuous at a.

Proof. Fix $t \in T$. Assume that $f$ is continuous at the point $a$ and denote $g(x)=f\left(a_{t}^{x}\right)$ for all $x \in X_{t}$. Then $\lim _{x \rightarrow a_{t}} g(x)=\lim _{x \rightarrow a_{t}} f\left(a_{t}^{x}\right)=f(a)=g\left(a_{t}\right)$. 
We argue similarly in the case $Y$ is a metric space and $f$ is strongly separately continuous.

Proposition 3.3. Let $X \subseteq \prod_{t \in T} X_{t}$ be an $\mathcal{S}$-open subset of a product of topological spaces $\left(X_{t}, \mathcal{T}_{t}\right)$ and let $\mathcal{T}$ be a topology on $X$. If one of the following conditions holds

(1) $(X, \mathcal{T})=(X, \tau)$, or

(2) $\left(X_{t}, \mathcal{T}_{t}\right)=(\mathbb{R}, \mathcal{E})$ for every $t \in T$ and $(X, \mathcal{T})$ is a topological vector space, then $\mathcal{T}$ is coordinated with $\left(\mathcal{T}_{t}: t \in T\right)$.

Proof. (1) It immediately implies from the definition of the Tychonoff topology.

(2) Fix $a=\left(a_{s}\right)_{s \in T} \in X$ and $t \in T$. Without loss of generality we may assume that $a_{s}=0$ for all $s \in S$. Let $b=1$. Then $a_{t}^{b} \in \sigma_{1}(a) \subseteq X$. Since the function $\varphi:(X, \mathcal{T}) \times \mathbb{R} \rightarrow(X, \mathcal{T}), \varphi(y, \lambda)=\lambda y$, is continuous, we have that $\lim _{\lambda \rightarrow 0} \varphi(y, \lambda)=0$ in $(X, \mathcal{T})$ for every $y \in X$. Then $\lim _{x \rightarrow 0} a_{t}^{x}=$ $\lim _{x \rightarrow 0} \varphi\left(a_{t}^{b}, x\right)=0$ in $(X, \mathcal{T})$.

Theorem 3.4. Let $X \subseteq \prod_{t \in T} X_{t}$ be an $\mathcal{S}$-open set and $(Y, d)$ be a metric space. A mapping $f:(X, \mathcal{S}) \rightarrow Y$ is continuous if and only if $f:(X, \mathcal{T}) \rightarrow Y$ is strongly separately continuous for an arbitrary topology $\mathcal{T}$ on $X$.

Proof. Necessity. Fix a topology $\mathcal{T}$ on $X$ and consider the partition $\left(\sigma\left(x_{i}\right)\right.$ : $i \in I)$ of the set $X$ on $\mathcal{S}$-components $\sigma\left(x_{i}\right)$. We notice that $\left.f\right|_{\sigma\left(x_{i}\right)}=y_{i}$, where $y_{i} \in Y$ for all $i \in I$, since $f$ is continuous on $(X, \mathcal{S})$. Let $a=\left(a_{t}\right)_{t \in T} \in X$ and $t \in T$. If $x \in X$, then $x \in \sigma\left(x_{i}\right)$ for some $i \in I$. Moreover, $x_{t}^{a} \in \sigma\left(x_{i}\right)$. Then $f(x)=f\left(x_{t}^{a}\right)=y_{i}$. Hence, $d\left(f(x), f\left(x_{t}^{a}\right)\right)=0$ for all $x \in X$. Hence, $f$ is strongly separately continuous on $(X, \mathcal{T})$.

Sufficiency. Put $\mathcal{T}=\mathcal{S}$. Fix $x_{0} \in X$ and show that $f$ is continuous at $x_{0}$ on $(X, \mathcal{S})$. Let $x_{0} \in \sigma\left(x_{i}\right)$ for some $i \in I$. Since $f$ is strongly separately continuous at $x_{0}$ and $\sigma\left(x_{0}\right)=\sigma\left(x_{i}\right)$, we have $d\left(f(x), f\left(x_{t}^{x_{0}}\right)\right)=0$ for all $x \in \sigma\left(x_{i}\right)$ and $t \in T$. Consequently, $f(x)=f\left(x_{0}\right)$ for all $x \in \sigma\left(x_{i}\right)$. Since the set $\sigma\left(x_{i}\right)$ is open in $(X, \mathcal{S}), f$ is continuous at $x_{0}$.

Now we give a necessary and sufficient condition for a strongly separately continuous mapping to be continuous.

Theorem 3.5. Let $X \subseteq \prod_{t \in T} X_{t}$ be an $\mathcal{S}$-open subset of a product of topological spaces $X_{t}, \mathcal{T}$ be a topology on $X$ such that $(X, \mathcal{T})$ is a locally projectively symmetric space, $(Y, d)$ be a metric space and let $f:(X, \mathcal{T}) \rightarrow Y$ be a strongly 
separately continuous mapping at the point $a=\left(a_{t}\right)_{t \in T} \in X$. Then $f$ is continuous at the point $a$ if and only if

$$
\begin{gathered}
\text { for every } \varepsilon>0 \text { there exist a set } T_{0} \subseteq T \text { with }\left|T_{0}\right|<\aleph_{0} \\
\text { and a neighborhood } U \text { of a in }(X, \mathcal{T}) \text { such that } \\
\qquad\left(f(a), f\left(x_{T_{0}}^{a}\right)\right)<\varepsilon \text { for all } x \in U .
\end{gathered}
$$

Proof. Necessity. Suppose $f$ is continuous at the point $a$ and $\varepsilon>0$. Take a neighborhood $U$ of $a$ such that $d(f(x), f(a))<\varepsilon$ for all $x \in U$ and put $T_{0}=\emptyset$. Then $x_{T_{0}}^{a}=x$, which implies condition (3.2).

Sufficiency. Fix $\varepsilon>0$. Using the condition of the theorem we take a finite set $T_{0} \subseteq T$ and a neighborhood $U$ of $a$ in $(X, \tau)$ such that

$$
d\left(f(a), f\left(x_{T_{0}}^{a}\right)\right)<\frac{\varepsilon}{2}
$$

for every $x \in U$. If $T_{0}=\emptyset$, then $d(f(x), f(a))<\varepsilon$ for all $x \in U$. Now assume $T_{0}=\left\{t_{1}, \ldots, t_{n}\right\}$. Since $f$ is strongly separately continuous at $a$, for every $k=1, \ldots, n$ we choose a neighborhood $V_{k}$ of the point $a$ such that

$$
d\left(f(x), f\left(x_{t_{k}}^{a}\right)<\frac{\varepsilon}{2 n}\right.
$$

for all $x \in V_{k}$. We take a projectively symmetric with respect to the point $a$ neighborhood $W$ of $a$ such that

$$
W \subseteq U \cap\left(\bigcap_{k=1}^{n} V_{k}\right)
$$

Since $W$ is projectively symmetric set with respect to $a$, we can show inductively that $x_{\left\{t_{1}, \ldots, t_{k}\right\}}^{a} \in W$ for every $k=1, \ldots, n$ and for every $x \in W$. Then for all $x \in W$ we have

$$
\begin{gathered}
d(f(x), f(a)) \leq d\left(f(x), f\left(x_{T_{0}}^{a}\right)\right)+d\left(f\left(x_{T_{0}}^{a}\right), f(a)\right)< \\
<d\left(f(x), f\left(x_{t_{1}}^{a}\right)\right)+d\left(f\left(x_{t_{1}}^{a}\right), f\left(x_{\left\{t_{1}, t_{2}\right\}}^{a}\right)\right)+\cdots+ \\
+d\left(f\left(x_{\left\{t_{1}, \ldots, t_{n-1}\right\}}^{a}\right), f\left(x_{\left\{t_{1}, \ldots, t_{n}\right\}}^{a}\right)\right)+\frac{\varepsilon}{2}< \\
<\frac{\varepsilon}{2 n} \cdot n+\frac{\varepsilon}{2}=\varepsilon .
\end{gathered}
$$

Hence, $f$ is continuous at the point $a$.

We notice that the similar condition given by Visnyai in [3] for functions defined on $\ell_{2}$ is stronger than condition (3.2).

The following corollary generalizes the result of Dzagnidze [1, Theorem 2.1].

Corollary 3.6. Let $X$ be an $\mathcal{S}$-open subset of a product $\prod_{t \in T} X_{t}$ of topological spaces $X_{t},|T|<\aleph_{0}$ and $(Y, d)$ be a metric space. Then any strongly separately continuous mapping $f:(X, \tau) \rightarrow Y$ is continuous. 
Proof. Fix an arbitrary point $a \in X$ and a strongly separately continuous mapping $f:(X, \tau) \rightarrow Y$. It is easy to see that $f$ satisfy condition (3.2). Indeed, for $\varepsilon>0$ we put $T_{0}=T$ and $U=X$. Then for all $x \in U$ we have $x_{T_{0}}^{a}=a$ and consequently $d\left(f(a), f\left(x_{T_{0}}^{a}\right)\right)=0<\varepsilon$. Hence, $f$ is continuous at $a$ by Theorem 3.5. provided $(X, \tau)$ is a locally projectively symmetric space.

The proposition below shows that Corollary 3.6 is not valid for a product of infinitely many topological spaces.

Proposition 3.7. Let $X=\prod_{t \in T} X_{t}$ be a product of topological spaces $X_{t}$, where $\left|X_{t}\right|>1$ for every $t \in T$, let $|T|>\aleph_{0}$ and $(Y, d)$ be a metric space with $|Y|>$ 1. Then there exists a strongly separately continuous everywhere discontinuous mapping $f:(X, \tau) \rightarrow Y$.

Proof. Fix $x_{0} \in X$ and $y_{1}, y_{2} \in Y, y_{1} \neq y_{2}$. According to Proposition 2.1. $\sigma\left(x_{0}\right) \neq \emptyset \neq X \backslash \sigma\left(x_{0}\right)$. Set $f(x)=y_{1}$ if $x \in \sigma\left(x_{0}\right)$ and $f(x)=y_{2}$ if $x \in$ $X \backslash \sigma\left(x_{0}\right)$. Then $f$ is everywhere discontinuous on $X$. Indeed, let $a \in X$ and $f(a)=y_{1}$. Take an open neighborhood $V$ of $y_{1}$ such that $y_{2} \notin V$. If $U$ is an arbitrary neighborhood of $a$ in $(X, \tau)$, then there is $x \in U \backslash \sigma\left(x_{0}\right)$. Therefore, $f(x)=y_{2} \notin V$ and $f$ is discontinuous at $a$. Similarly, $f$ is discontinuous at $a$ in the case $f(a)=y_{2}$.

Since the set $\sigma\left(x_{0}\right)$ is clopen in $(X, \mathcal{S})$, the mapping $f:(X, \mathcal{S}) \rightarrow Y$ is continuous. It remains to apply Theorem 3.4 .

\section{A NECESSARY CONDItION ON THE DISCONTINUITY POINT SET}

Let $f: X \rightarrow Y$ a mapping between topological spaces. By $C(f)$ we denote the continuity point set of $f$ and by $D(f)$ we denote the discontinuity point set of $f$.

Let $\mathcal{U}_{x}$ be a system of all neighborhoods of a point $x$ in $X$. For a mapping $f: X \rightarrow(Y, d)$ we set

$$
\omega_{f}(A)=\sup _{x^{\prime}, x^{\prime \prime} \in A} d\left(f\left(x^{\prime}\right), f\left(x^{\prime \prime}\right)\right) \quad \text { and } \quad \omega_{f}(x)=\inf _{U \in \mathcal{U}_{x}} \omega_{f}(U) .
$$

Definition 4.1. A set $W \subseteq \sigma(a)$ is called nearly open in $(\sigma(a), \tau)$ if for any finite set $T_{0} \subseteq T$ the set

$$
W_{T_{0}}=\left\{z \in \prod_{t \in T_{0}} X_{t}: a_{T_{0}}^{z} \in W\right\}
$$

is open in the space $\left(\prod_{t \in T_{0}} X_{t}, \tau\right)$.

Theorem 4.1. Let $\left(X_{t}: t \in T\right)$ be a family of topological spaces, $X=\prod_{t \in T} X_{t}$, $a=\left(a_{t}\right)_{t \in T} \in X,(Y, d)$ be a metric space and $f:(\sigma(a), \tau) \rightarrow Y$ be a strongly 
separately continuous mapping. Then the discontinuity point set $D(f)$ of $f$ is nearly open in $(\sigma(a), \tau)$.

Proof. Let $T_{0} \subseteq T$ be an arbitrary finite set and $Z=\prod_{t \in T_{0}} X_{t}$. For $z \in Z$ we write $\varphi(z)=a_{T_{0}}^{z}$ and $G=(D(f))_{T_{0}}$.

If $T_{0}=\emptyset$, then $G=\emptyset$. Now let $T_{0}=\left\{t_{1}, \ldots, t_{n}\right\}, w=\left(w_{t}\right)_{t \in T_{0}} \in G$, $u=\varphi(w) \in \sigma(a)$ and $\varepsilon=\frac{1}{3} \omega_{f}(u)$. We observe that $\varepsilon>0$, provided $f$ is discontinuous at $u$. Since $f$ is strongly separately continuous at the point $u$, there exists a basic neighborhood $U_{0}$ of $u$ in $(X, \tau)$ such that

$$
d\left(f(x), f\left(x_{\{t\}}^{u}\right)\right)<\frac{\varepsilon}{6 n}
$$

for all $t \in T_{0}$ and $x \in U_{0} \cap \sigma(a)$. Since the mapping $\varphi:(Z, \tau) \rightarrow(\sigma(a), \tau)$ is continuous, there exists a basic neighborhood $W_{0}$ of $w$ in $(Z, \tau)$ such that $\varphi\left(W_{0}\right) \subseteq U_{0}$.

We show that

$$
d\left(f(x), f\left(x_{T_{0}}^{\varphi(z)}\right)\right)<\frac{\varepsilon}{3}
$$

for any $z \in W_{0}$ and $x \in U_{0} \cap \sigma(a)$. Let $x_{0}=x$ and $x_{k}=\left(x_{k-1}\right)_{t_{k}}^{\varphi(z)}, k=1, \ldots, n$. Then $x_{n}=x_{T_{0}}^{\varphi(z)}$. Moreover, since $x, \varphi(z) \in U_{0}, x_{k} \in U_{0}$ for every $k$. It follows from (4.1) that

$$
d\left(f\left(x_{k-1}\right), f\left(\left(x_{k-1}\right)_{t_{k}}^{u}\right)\right)<\frac{\varepsilon}{6 n} \quad \text { i } \quad d\left(f\left(x_{k}\right), f\left(\left(x_{k}\right)_{t_{k}}^{u}\right)\right)<\frac{\varepsilon}{6 n}
$$

for every $k=1, \ldots, n$. Taking into account the equality $\left(x_{k-1}\right)_{t_{k}}^{u}=\left(x_{k}\right)_{t_{k}}^{u}$, we obtain

Hence,

$$
d\left(f\left(x_{k-1}\right), f\left(x_{k}\right)\right)<\frac{\varepsilon}{3 n} .
$$

$$
d\left(f(x), f\left(x_{T_{0}}^{\varphi(z)}\right)\right)=d\left(f\left(x_{0}\right), f\left(x_{n}\right)\right) \leq \sum_{k=1}^{n} d\left(f\left(x_{k-1}\right), f\left(x_{k}\right)\right)<\frac{\varepsilon}{3} .
$$

Now we prove that

$$
\omega_{f}(\varphi(z)) \geq \frac{\varepsilon}{3}
$$

for all $z \in W_{0}$. Let $z \in W_{0}$ and $x=\varphi(z)$. Since $\omega_{f}(u)=3 \varepsilon$, there exists a net $\left(u_{\lambda}\right)_{\lambda \in \Lambda}$ of points of $\sigma(a) \cap U_{0}$ such that $u_{\lambda} \underset{\lambda \in \Lambda}{\rightarrow} u$ in $(X, \tau)$ and $d\left(f\left(u_{\lambda}\right), f(u)\right) \geq \varepsilon$ for every $\lambda \in \Lambda$. We notice that $u_{T_{0}}^{x}=x$. Therefore, $v_{\lambda}=\left(u_{\lambda}\right)_{T_{0}}^{x} \underset{\lambda \in \Lambda}{\longrightarrow} x$. It follows from (4.2) that

$$
d(f(u), f(x))<\frac{\varepsilon}{3} \quad \mathrm{i} \quad d\left(f\left(u_{\lambda}\right), f\left(v_{\lambda}\right)\right)<\frac{\varepsilon}{3} .
$$


Hence,

$$
d\left(f(x), f\left(v_{\lambda}\right)\right) \geq d\left(f(u), f\left(u_{\lambda}\right)\right)-d(f(u), f(x))-d\left(f\left(u_{\lambda}\right), f\left(v_{\lambda}\right)\right)>\frac{\varepsilon}{3},
$$

consequently, $\omega_{f}(x) \geq \frac{\varepsilon}{3}$. Therefore, $x \in D(f)$. Thus, $W_{0} \subseteq G$, which implies that $G$ is open in $(Z, \tau)$.

\section{A SUFFICIENT CONDITION ON THE DisCONTINUITY POINT SET}

The definition of strongly separately continuous mapping easily implies the following properties.

Proposition 5.1. Let $X$ be an $\mathcal{S}$-open subset of a product $\prod_{t \in T} X_{t}$ of a family of topological spaces $X_{t}$ and $\mathcal{T}$ be a topology on $X$.

If $f, g:(X, \mathcal{T}) \rightarrow \mathbb{R}$ are strongly separately continuous mappings at $x_{0} \in X$, then the mappings $f(x) \pm g(x), f(x) \cdot g(x),|f(x)|, \min \{f(x), g(x)\}, \max \{f(x), g(x)\}$ are strongly separately continuous at $x_{0}$.

If $f(x)=\sum_{n=1}^{\infty} f_{n}(x)$ is a sum of a uniformly convergent series of strongly separately continuous at $x_{0} \in X$ mappings $f_{n}:(X, \mathcal{T}) \rightarrow \mathbb{R}$, then $f$ is strongly separately continuous at $x_{0}$.

If $(X,\|\cdot\|)$ is a normed space, $a \in X$ and $r>0$, then we write

$$
B(a, r)=\{x \in X:\|x-a\|<r\} \quad \text { and } \quad B[a, r]=\{x \in X:\|x-a\| \leq r\} .
$$

Theorem 5.2. Let $\left(\left(X_{n},\|\cdot\|_{n}\right)\right)_{n=1}^{\infty}$ be a sequence of normed spaces, $a \in \prod_{n=1}^{\infty} X_{n}$, $w=\left(w_{n}\right)_{n=1}^{\infty} \in \sigma(a),\left(r_{n}\right)_{n=1}^{\infty}$ be a sequence of positive reals and

$$
W=\left(\prod_{n=1}^{\infty} B\left(w_{n}, r_{n}\right)\right) \cap \sigma(a) .
$$

Then there exists a strongly separately continuous function $f:(\sigma(a), \tau) \rightarrow[0,1]$ such that

$$
W=D(f) \subseteq f^{-1}(0) .
$$

Proof. Assume without loss of generality that $a=(0,0, \ldots)$. We put $X=$ $(\sigma(a), \tau)$ and for every $n \in \mathbb{N}$ let

$$
\begin{aligned}
& B_{n}=B\left(w_{n}, r_{n}\right), \quad Y_{n}=X_{1} \times \cdots \times X_{n} \text { and } \\
& d_{n}(x, y)=\max _{1 \leq i \leq n}\left\|x_{i}-y_{i}\right\|_{i} \text { for all } x, y \in Y_{n} .
\end{aligned}
$$

If $x=\left(x_{n}\right)_{n=1}^{\infty} \in X$ and $k \in \mathbb{N}$, then $p_{k}(x)$ stands for $\left(x_{1}, \ldots, x_{k}\right)$.

For every $x=\left(x_{n}\right)_{n=1}^{\infty} \in X$ we set $h(x)=\left(\frac{1}{r_{n}}\left(x_{n}-w_{n}\right)\right)_{n=1}^{\infty}$. Then $h: X \rightarrow X$ is a homeomorphism. 
For every $n \in \mathbb{N}$ define

$$
\begin{gathered}
B_{n}^{\prime}=\left\{x \in X_{n}:\|x\|_{n}<1\right\}, \quad S_{n}=\left\{x \in X_{n}:\|x\|_{n}=1\right\}, \\
A_{1}=X_{1} \backslash B_{1}^{\prime}, \quad A_{n}=\prod_{i=1}^{n-1} B_{i}^{\prime} \times\left(X_{n} \backslash B_{n}^{\prime}\right) \text { if } n \geq 2, \\
C_{n}=\left(A_{n} \times \prod_{i=n+1}^{\infty} X_{i}\right) \cap X, \quad C=\bigsqcup_{n=1}^{\infty} C_{n} .
\end{gathered}
$$

We observe that

$$
W^{\prime}=h(W)=\left(\prod_{n=1}^{\infty} B_{n}^{\prime}\right) \cap X \quad \text { and } \quad C=X \backslash W^{\prime} .
$$

Now for every $n \in \mathbb{N}$ we consider a function $f_{n}: X \rightarrow \mathbb{R}$,

$$
f_{n}(x)=d_{n}\left(p_{n}(x), Y_{n} \backslash A_{n}\right),
$$

and for all $x \in X$ set

$$
g(x)= \begin{cases}f_{n}(x), & \text { if } p_{n}(x) \in A_{n} \text { for some } n \in \mathbb{N}, \\ 0, & \text { otherwise. }\end{cases}
$$

Claim 1. The function $g: X \rightarrow \mathbb{R}$ is strongly separately continuous.

Proof. Fix $u \in X$ and $k \in \mathbb{N}$. For $x \in X$ we write $y=x_{k}^{u}$ and estimate the difference $|g(x)-g(y)|$. If $p_{n}(x) \in A_{n}$ and $p_{m}(y) \in A_{m}$ for some $n, m \in \mathbb{N}$, then, using the equality $f_{n}(y)=f_{m}(x)=0$ in the case $n \neq m$, we obtain

$$
\begin{aligned}
& |g(x)-g(y)| \leq\left|f_{n}(x)-f_{n}(y)\right|+\left|f_{m}(x)-f_{m}(y)\right| \leq \\
\leq & \left|d_{n}\left(p_{n}(x), p_{n}(y)\right)\right|+\left|d_{m}\left(p_{m}(x), p_{m}(y)\right)\right| \leq 2\left\|x_{k}-u_{k}\right\|_{k} .
\end{aligned}
$$

If $p_{n}(x) \in A_{n}$ for some $n$ and $y \notin C$, then $g(y)=f_{n}(y)=0$ and

$$
|g(x)-g(y)| \leq\left|d_{n}\left(p_{n}(x), p_{n}(y)\right)\right| \leq\left\|x_{k}-u_{k}\right\|_{k} .
$$

The same estimation is valid if $x \notin C$ and $y \in C$. Finally, if $x, y \notin C$, then $|g(x)-g(y)|=0$. Hence, for all $x \in X$ we have

$$
|g(x)-g(y)| \leq 2\left\|x_{k}-u_{k}\right\|_{k},
$$

which implies that

$$
0 \leq \lim _{x \rightarrow u}|g(x)-g(y)| \leq 2 \lim _{x \rightarrow u}\left\|x_{k}-u_{k}\right\|_{k}=0 .
$$

Therefore, $g$ is strongly separately continuous at $u$.

Claim 2. The equality

$$
d_{n}\left(u, Y_{n} \backslash A_{n}\right)=\min _{1 \leq i \leq n}\left\|u_{i}-S_{i}\right\|_{i} .
$$

holds for all $n \in \mathbb{N}$ and $u=\left(u_{1}, \ldots, u_{n}\right) \in A_{n}$. 
Proof. Fix $n \in \mathbb{N}, u=\left(u_{1}, \ldots, u_{n}\right) \in A_{n}$ and let $B=Y_{n} \backslash A_{n}, \rho_{i}=\left\|u_{i}-S_{i}\right\|_{i}$ if $i=1, \ldots, n$, and $\rho=\min _{1 \leq i \leq n} \rho_{i}$.

We show that $d_{n}(u, B) \geq \rho$. If $\left\|u_{n}\right\|_{n}=1$, then $\rho_{n}=0=\rho$ and the inequality is obvious. Suppose $\left\|u_{n}\right\|_{n}>1$, choose any $y=\left(y_{1}, \ldots, y_{n}\right)$ with $d_{n}(u, y)<\rho$ and check that $y \in A_{n}$. Notice that $\left\|u_{i}-y_{i}\right\|_{i}<\rho_{i}$ for every $i=1, \ldots, n$. Assume that there exists $1 \leq i \leq n-1$ such that $\left\|y_{i}\right\|_{i} \geq 1$. Then there exists $b \in S_{i}$ such that $b=u_{i}+t\left(y_{i}-u_{i}\right)$ for some $t \in(0,1]$. Then $\left\|u_{i}-b\right\|_{i}=t\left\|u_{i}-y_{i}\right\|_{i}<\rho_{i}$, a contradiction. Hence, $y_{i} \in B_{i}^{\prime}$ for every $i=1, \ldots, n-1$. Similarly we can show that $y_{n} \notin B_{n}^{\prime}$. Hence, $y \in A_{n}$.

Now we show that $d_{n}(u, B) \leq \rho$. Fix $\varepsilon>0$ and assume that $\rho=\rho_{i}$ for some $1 \leq i \leq n$. If $i<n$, then there exists $v_{i} \in S_{i}$ with $\left\|u_{i}-v_{i}\right\|_{i}<\rho+\varepsilon$. If $i=n$, then, since $\left\|u_{n}-S_{n}\right\|_{n}=\left\|u_{n}-B_{n}^{\prime}\right\|_{n}$, there exists $v_{n} \in B_{n}^{\prime}$ such that $\left\|u_{n}-v_{n}\right\|_{n}<\rho+\varepsilon$. Let $v_{j}=u_{j}$ for $j \neq i$. Then $v=\left(v_{1}, \ldots, v_{n}\right) \in B$ and $d_{n}(u, v)<\rho+\varepsilon$, which implies that $d_{n}(u, B) \leq \rho$.

Therefore, $d_{n}(u, B)=\rho$.

Claim 3. $W^{\prime} \subseteq D(g)$.

Proof. Fix $u=\left(u_{1}, \ldots, u_{n}, 0, \ldots\right) \in W^{\prime}$. For every $i=1, \ldots, n$ we set $\rho_{i}=$ $\left\|u_{i}-S_{i}\right\|_{i}$ and $\rho=\min _{1 \leq i \leq n} \rho_{i}$. Then $\rho \in(0,1]$. For every $i \geq n+1$ we choose $x_{i} \in X_{i}$ such that $\left\|x_{i}\right\|_{i}=1+\rho$ and consider a sequence $\left(x^{m}\right)_{m=1}^{\infty}$ such that

$$
x^{m}=\left(u_{1}, \ldots, u_{n}, 0, \ldots, 0, x_{m+n}, 0, \ldots\right) .
$$

Clearly, $x^{m} \in C_{m+n}$ and $x^{m} \rightarrow u$. Since

$$
\left\|x_{m+n}-z\right\|_{m+n} \geq\left|\left\|x_{m+n}\right\|_{m+n}-\|z\|_{m+n}\right|=\rho
$$

for all $z \in S_{m+n}$,

$$
g\left(x^{m}\right)=\min \left\{\rho,\left\|x_{m+n}-S_{m+n}\right\|_{m+n}\right\}=\rho .
$$

Hence, $\lim _{m \rightarrow \infty} g\left(x^{m}\right)=\rho>0=g(u)$, which implies the discontinuity of $g$ at $u$.

Claim 4. $C \subseteq C(g)$.

Proof. Fix $u=\left(u_{n}\right)_{n=1}^{\infty} \in C$ and $\varepsilon>0$. Let $p_{n}(u) \in A_{n}$ for some $n \in \mathbb{N}$ and consider the case $\left\|u_{n}\right\|_{n}>1$. Since $\psi: Y_{n} \rightarrow \mathbb{R}, \psi\left(x_{1}, \ldots, x_{n}\right)=\min _{1 \leq i \leq n}\left\|x_{i}-S_{i}\right\|_{i}$, is continuous at $p=p_{n}(u)$, there exists a neighborhood $U=U_{1} \times \cdots \times U_{n}$ of $p$ in $Y_{n}$ such that $|\psi(x)-\psi(u)|<\varepsilon$ for all $x \in U$. Let

$$
G=\prod_{i=1}^{n-1}\left(U_{i} \cap B_{i}^{\prime}\right) \times\left(U_{n} \cap\left(X_{n} \backslash \overline{B_{n}^{\prime}}\right)\right) \times \prod_{i=n+1}^{\infty} X_{i} .
$$

Then $|g(x)-g(u)|=|\psi(x)-\psi(u)|<\varepsilon$ for all $x \in G \cap X$. 
Now suppose $\left\|u_{n}\right\|_{n}=1$. Then $g(u)=f_{n}(u)=0$. Set

$$
V=\prod_{i=1}^{n-1} B_{i}^{\prime} \times B\left(u_{n}, \varepsilon\right) \times \prod_{i=n+1}^{\infty} X_{i} .
$$

Let $x \in V \cap X$. If $\left\|x_{n}\right\|_{n} \leq 1$, then $g(x)=0$, and if $\left\|x_{n}\right\|_{n}>1$, then

$$
g(x)=\min _{1 \leq i \leq n}\left\|x_{i}-S_{i}\right\|_{i} \leq\left\|x_{n}-S_{n}\right\|_{n} \leq\left\|x_{n}-u_{n}\right\|_{n}<\varepsilon .
$$

Hence, $|g(x)-g(u)|<\varepsilon$ for all $x \in V \cap X$.

Therefore, $u \in C(g)$.

Claim 3 and Claim 4 imply that $D(g)=W^{\prime}$. Moreover, $g(x)=0$ for all $x \in W^{\prime}$.

Consider the function $\varphi: X \rightarrow \mathbb{R}$ such that $\varphi(x)=g(h(x))$ for all $x \in X$.

Fix $n \in \mathbb{N}$ and $u \in X$. Then $h\left(x_{n}^{u}\right)=(h(x))_{n}^{h(u)}$ for every $x \in X$. We have

$$
\begin{gathered}
\lim _{x \rightarrow u}\left|\varphi(x)-\varphi\left(x_{n}^{u}\right)\right|=\lim _{x \rightarrow u}\left|g(h(x))-g\left(h\left(x_{n}^{u}\right)\right)\right|= \\
=\lim _{h(x) \rightarrow h(u)}\left|g(h(x))-g\left((h(x))_{n}^{h(u)}\right)\right|=0,
\end{gathered}
$$

since $g$ is strongly separately continuous at $h(u)$ with respect to the $n$-th variable. Hence, $\varphi: X \rightarrow \mathbb{R}$ is strongly separately continuous.

If is easy to see that $\varphi$ is continuous at $x \in X$ if and only if $g$ is continuous at $h(x) \in X$. Hence, $D(\varphi)=h^{-1}(D(g))=h^{-1}\left(W^{\prime}\right)=W$. Moreover, $\varphi^{-1}(0)=$ $h^{-1}\left(g^{-1}(0)\right) \supseteq h^{-1}\left(W^{\prime}\right)=W$.

Finally, for every $x \in X$ we put

$$
f(x)=\min \{\varphi(x), 1\} .
$$

Note that $D(f)=D(\varphi)=W$ and $f(x)=0$ for all $x \in W$.

It remains to observe that $f: X \rightarrow[0,1]$ is strongly separately continuous by Proposition 5.1

Theorem 5.3. Let $\left(\left(X_{n},\|\cdot\|_{n}\right)\right)_{n=1}^{\infty}$ be a sequence of finite-dimensional normed spaces, $a \in \prod_{n=1}^{\infty} X_{n}$ and $W \subseteq \sigma(a)$ be a nearly open set. Then there exists a strongly separately continuous function $f:(\sigma(a), \tau) \rightarrow[0,1]$ such that $D(f)=W$.

Proof. Without loss of generality we can assume that $a=(0,0, \ldots)$. For every $n \in \mathbb{N}$ let

$$
\begin{aligned}
Y_{n} & =X_{1} \times \cdots \times X_{n}, \quad Z_{n}=Y_{n} \times\{0\} \times\{0\} \times \ldots, \\
G_{n} & =\left\{\left(x_{1}, \ldots, x_{n}\right) \in Y_{n}:\left(x_{1}, \ldots, x_{n}, 0, \ldots\right) \in W\right\},
\end{aligned}
$$

and $X=(\sigma(0), \tau)$. 
Let $x=\left(x_{n}\right)_{n=1}^{\infty}$ be an arbitrary point of $W$ such that $x_{n}=0$ for all $n>N$. Since $W$ is nearly open, for every $k=1, \ldots, N$ there exists $r_{k}(x)>0$ such that $F_{1}=\prod_{k=1}^{N} B\left[x_{k}, r_{k}(x)\right] \subseteq G_{N}$. Since compact set $K_{1}=F_{1} \times\{0\}$ is contained in the open in $Y_{N+1}$ set $G_{N+1}$, we can find $r_{N+1}(x)>0$ such that

$$
F_{2}=F_{1} \times B\left[0, r_{N+1}(x)\right] \subseteq G_{N+1} .
$$

Now the compactness of $K_{2}=F_{2} \times\left.\{0\} \subseteq W\right|_{Y_{N+2}}$ implies that there exists $r_{N+2}(x)>0$ such that

$$
F_{3}=F_{2} \times B\left[0, r_{N+2}(x)\right] \subseteq G_{N+2} .
$$

By repeating this process, we obtain a sequence $\left(r_{n}(x)\right)_{n=1}^{\infty}$ of positive reals such that

$$
\left(\prod_{n=1}^{\infty} B\left[x_{n}, r_{n}(x)\right]\right) \cap X \subseteq W .
$$

Now let $W(x)=\left(\prod_{n=1}^{\infty} B\left(x_{n}, r_{n}(x)\right)\right) \cap X$.

Hence, $W=\bigcup_{x \in W} W(x)$. Since for every $n \in \mathbb{N}$ the family $\left(W(x) \cap Z_{n}: x \in W\right)$ forms an open covering of $V_{n}=W \cap Z_{n}$ in $Z_{n}$, there exists a countable set $I_{n} \subseteq W$ such that the family $\left(W(x) \cap Z_{n}: x \in I_{n}\right)$ is a covering of $V_{n}$. Put $I=\bigcup_{n=1}^{\infty} I_{n}$. Then

$$
W=\bigcup_{n=1}^{\infty} V_{n}=\bigcup_{n=1}^{\infty} \bigcup_{x \in I_{n}}\left(W(x) \cap Z_{n}\right)=\bigcup_{x \in I} W(x) .
$$

Let $I=\left\{x_{m}: m \in \mathbb{N}\right\}$ and $W_{m}=W\left(x_{m}\right)$.

According to Theorem 5.2 there exists a sequence $\left(f_{m}\right)_{m=1}^{\infty}$ of strongly continuous functions $f_{m}: X \rightarrow[0,1]$ such that $D\left(f_{m}\right)=W_{m} \subseteq f_{m}^{-1}(0)$. The last inclusion implies that every $f_{m}$ is lower semi-continuous on $X$. For all $x \in X$ we define

$$
f(x)=\sum_{m=1}^{\infty} \frac{1}{2^{m}} f_{m}(x) .
$$

Then Proposition 5.1 implies that $f: X \rightarrow[0,1]$ is strongly separately continuous function. Moreover, $f$ is lower semi-continuous as a sum of uniformly convergent series of lower semi-continuous functions.

Taking into account that $D\left(g_{1}+g_{2}\right)=D\left(g_{1}\right) \cup D\left(g_{2}\right)$ for any two lower semicontinuous functions $g_{1}$ and $g_{2}$, we obtain

$$
D(f)=\bigcup_{m=1}^{\infty} D\left(f_{m}\right)=W
$$


Combining Theorems 4.1 and 5.3 we obtain

Theorem 5.4. Let $X=\prod_{n=1}^{\infty} X_{n}$ be a product of finite-dimensional normed spaces $X_{n}, a \in X$ and $W \subseteq \sigma(a)$. Then $W$ is the discontinuity point set of some strongly separately continuous function $f:(\sigma(a), \tau) \rightarrow \mathbb{R}$ if and only if $W$ is nearly open in $(\sigma(a), \tau)$.

\section{REFERENCES}

[1] DZAGNIDZE, O. Separately continuous function in a new sense are continuous, Real Anal. Exch. 24 (1998-99), 695-702.

[2] ČINČURA, J.-ČALÁT, T.-VISNYAI, T. On separately continuous functions $f: \ell^{2} \rightarrow$ $\mathbb{R}$, Acta Acad. Paedagog. Agriensis, XXXI (2004), 11-18.

[3] VISNYAI, T. Strongly separately continuous and separately quasicontinuous functions $f: \ell^{2} \rightarrow \mathbb{R}$, Real Anal. Exch. 38 (2) (2013), 499-510.

* Chernivtsi National University

KOTSYUBYNS'KOHO 2

58012 CHERNIVTIS

UKRAINE

E-mail address: maslenizza.ua@gmail.com

** Chernivtsi National University

KOTSYUBYNS'Kоно 2

58012 ChERNIVTIS

UKRAINE

E-mail address: vmykhaylyuk@ukr.net 